\title{
Characterization of raloxifene as potential pharmacological agent against SARS-CoV-2
} and its variants

Authors:

Daniela Iaconis ${ }^{1}$, Carmine Talarico ${ }^{1}$, Candida Manelfi ${ }^{2}$, Maria Candida Cesta $^{2}$, Mara Zippoli ${ }^{1}$ Francesca Caccuri ${ }^{3}$, Giulia Matusali ${ }^{4}$, Licia Bordi ${ }^{4}$, Laura Scorzolini ${ }^{4}$, Enrico Bucci ${ }^{5}$, Arnaldo Caruso $^{3}$, Emanuele Nicastri ${ }^{4}$, Marcello Allegretti ${ }^{2}$, Andrea Rosario Beccari ${ }^{1}$

${ }^{1}$ Dompé Farmaceutici SpA, Via Tommaso De Amicis, 95, Napoli, Italy

${ }^{2}$ Dompé Farmaceutici SpA, Via Campo di Pile, L'Aquila, Italy

${ }^{3}$ Department of Molecular and Translational Medicine, Section of Microbiology and Virology, University of Brescia Medical School, Brescia, Italy.

${ }^{4}$ Lazzaro Spallanzani National Institute for Infectious Diseases, IRCCS Via Portuense 292, 00149 Rome, Italy

${ }^{5}$ Sbarro Health Research Organization, Biology Department CFT, Temple University, Philadelphia, USA

*Correspondence to: marcello.allegretti@dompe.com

Running title: Raloxifene as new pharmacological agent against COVID-19

The new coronavirus that emerged, called SARS-CoV-2, is the causative agent of the COVID-19 pandemic. The identification of potential drug candidates that can rapidly enter clinical trials for the prevention and treatment of COVID-19 is an urgent need, despite the recent introduction of several new vaccines for the prevention and protection of this infectious disease, which in many cases becomes severe. Drug repurposing (DR), a process for studying existing pharmaceutical products for new therapeutic indications, represents one of the most effective potential strategies employed to increase the success rate in the development of new drug therapies. We identified raloxifene, a known Selective Estrogen Receptor Modulator (SERM), as a potential pharmacological agent for the treatment of COVID-19 patients. Following a virtual screening campaign on the most relevant viral protein targets, in this work we report the results of the first pharmacological characterization of raloxifene in relevant cellular models of COVID-19 infection. The results obtained on all the most common viral variants originating in Europe, United Kingdom, Brazil, South Africa and India, currently in circulation, are also reported, confirming the efficacy of raloxifene and, consequently, the relevance of the proposed approach. 
Taken together, all the information gathered supports the clinical development of raloxifene and confirms that the drug can be proposed as a viable new option to fight the pandemic in at least some patient populations. The results obtained so far have paved the way for a first clinical study to test the safety and efficacy of raloxifene, just concluded in patients with mild to moderate COVID-19.

Keywords: COVID-19, drug repurposing, raloxifene, estrogen receptors, antiviral activity, Vero E6 cells, Calu-3 cells, SARS-CoV-2 viral variants.

\section{Introduction}

Coronaviruses are the causative agent of multiple respiratory and intestinal infection in humans and animals [1-3]. Unlike most other human coronaviruses, which only rarely cause severe disease and death [4], SARS-CoV-2 is able to cause severe acute respiratory illness, multi-organ failure and death, sharing common pathogenetic mechanisms with SARS-CoV and MERS-CoV betacoronavirus [5]. Common symptoms of COVID-19 include fever, sore throat, fatigue, cough, shortness of breath and dyspnea that may eventually progress towards acute respiratory distress syndrome, with the involvement of other systems/organs (e.g., heart, liver and kidneys) [6, 7] and up to the death in the most critical cases. About $80 \%$ of patients have mild to moderate disease, $14 \%$ have severe disease, and $6 \%$ become critical (namely, they develop respiratory failure, septic shock, and/or multiple organ

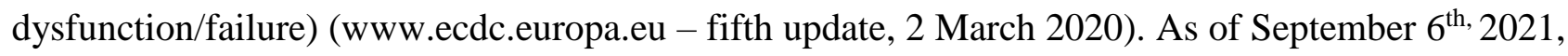
SARS-CoV-2 infection led to more than 4,5 million deaths worldwide (https://covid19. who.int/). To date, notwithstanding the advent of vaccine programs and constant social distancing interventions, it is believed that the virus is likely or very likely to become endemic $[8,9]$. In addition, the emerging of SARS-CoV-2 variants raises great concern for vaccine efficacy, reinfection events and increased transmissibility and disease severity. As the virus started to spread around the world, a mutated spike SARS-CoV-2 variant (D614G) emerged and was associated with increased infectivity, becoming the predominant variant in Europe and worldwide without any increase in disease severity [10-13]. In recent months, other variants were defined as "variants of concern" (VOC). The most relevant also from a clinical point of view are: B.1.1.7 (UK), B.1.351 (South African), B.1.1.28 (Brazilian P.1), B.1.427 and B.1.429 (Californian, also named CAL.20C), characterized by increased transmissibility, immune evasion and higher virulence [14-20]. As of May $11^{\text {th }}, 2021$, the so called "Indian" Delta variant (Delta B.1.617.2) was added to the WHO list of VOC; this variant seems to be able to escape 
adaptive immunity induced by prior wild type infection roughly half of the time and to be more infectious (around 60\%) than wild type SARS-CoV-2 [21]. The growing relevance of the rapidly emerging SARS-CoV-2 variants deserves further investigations, and new impetus will have to be given to research to increase the availability of broad-spectrum drugs or vaccines for long-term prevention, treatment and control of COVID-19, with the final goal to find new interventions and cures to complement vaccine programs. To identify potential therapeutic targets, one of the main studied mechanisms is the virus entry machinery, and several preclinical and clinical trials are ongoing to find new inhibitors of clinical relevance [22]. The entry machinery involves two key host proteins: the angiotensin-converting enzyme 2 (ACE2) and the cell surface transmembrane protease serine 2 (TMPRSS2) [23, 24]. In addition, also Neuropilin1 (NRP-1) has been recognized as an important receptor whose inhibition reduces SARS-CoV-2 entry and infectivity [25, 26].

Moreover, recent evidence shows that Nuclear Receptors (NRs), and in particular the sex hormone receptors, like estrogen and androgen receptors, could be involved in the outcome of COVID-19. These receptors regulate the viral entry protein expression and activity [27-29]. Additionally, a protective effect of estrogens in the progression of COVID-19 infection has been associated with their role in regulation of innate and adaptive immune responses, as well as in the control of the cytokine storm [30-34], whereas activation of androgen receptors seems to correlate with the worse COVID19 clinical outcome observed in men compared to women [29, 35-37].

Recently, several molecules with potential efficacy against SARS-CoV-2 were selected from an extensive virtual screening campaign based on the EXaSCale smArt pLatform Against paThogEns [38], a powerful tool for repurposing of drugs and compounds in new indications [39-42] for immediate response and quick identification of effective treatments, useful during pandemic situations. So, in the context of the Horizon 2020 project EXSCALATE4CoV, raloxifene, a wellknown SERM (Selective Estrogen Receptor Modulator) [43-47] was selected through an integrated approach of drug repurposing and in silico screening on SARS-CoV-2 target proteins, an approach that, combined with the scientific rationale and literature evidence that support a potential antiviral and protective action of SERMs in COVID-19, led the molecule to be selected as clinical candidate for studies in mild to moderate COVID-19 patients [38].

Raloxifene is a drug registered in Europe and US for the treatment and prevention of osteoporosis in postmenopausal women, and for the reduction of the risk of invasive breast cancer in postmenopausal women $[48,49]$. It is known to act as an agonist in the bone, liver and cardiovascular system and as antagonist in human breast and uterine tissues [50-52], and tissue specificity is relevant for its use in 
postmenopausal osteoporosis and prevention of breast cancer without increase of risk of endometrial cancer, differently from the behavior of other SERMs like tamoxifen $[53,54]$. The drug has also been studied in men for uses such as for treatment of schizophrenia, prostate cancer and osteoporosis [5557]. Recently, raloxifene has been also characterized in viral infections. It was found active against Ebola virus [58, 59], Hepatitis C virus [60, 61], Hepatitis B virus [62], and Zika virus [63]. Further, it showed efficacy in human female cells from nasal epithelium, against the Influenza Virus A [64], and as adjuvant antiviral treatment of chronic hepatitis $\mathrm{C}$ (CHC) in postmenopausal women [65]. These observations, together with raloxifene activity on the Estrogen Receptor (ER) pathways, highlight a possible relationship between clinical outcome and sex and age of patients with viral infections.

In this paper, we report for the first time a full characterization of the antiviral activity of raloxifene in two different well-established cellular contexts (Vero E6 and Calu-3) and we tested the potential influence of the most common COVID-19 variants on raloxifene biological activity. Raloxifene in vitro activity was high and consistent in the different cell lines tested, preserved in all main VOCs of clinical relevance.

Repurposing and in silico/experimental synergy are powerful useful approaches in case of pandemic infections by viruses and other pathogens, where an immediate response and the swift identification of effective treatments are of paramount importance. Taken together, the collected evidence confirms the potential of raloxifene as a promising agent with the potential to control COVID-19 infection with pleiotropic mechanisms supporting the rationale for the ongoing clinical investigation (study RLX0120, EudraCT Nr: 2020-003936-25) for the treatment of mild to moderate COVID-19 patients.

\section{Materials and methods}

\section{System Biology Screening}

First, we isolated 12 genes identified by The Host Genetic Initiative (https://www.covid19hg.org/results/r3/ ) as relevant for the infection and present in the data release number 3 (July 2020), namely: ANKRD32, CDRT4, PSMD13, ERO1L, LZTFL1, XCR1, FYCO1, IFNAR2, CXCR6, CCR9, AP000295.9, AK5. Based on a lookup of previous GWAS results in the GWAS ATLAS database (a database of publicly available GWAS summary statistics), these genes are considered primarily implicated in immunological phenotypes. Then, we looked at the human- 
SARS-CoV-2 interactome network as published [66], and extracted all the human genes included in the set. A list combining the two dataset was used as seeding for a BioGrid search by mean of Cytoscape v.3.8.0; the resulting enriched functional network connected those human proteins, known to directly bind SARS-CoV-2 proteins, with the human gene products involved in the host pathology. Subsequently, we screened 8721 Scopus-derived documents, referred to raloxifene, for the presence of at least one of the proteins/genes included in the Cytoscape-generated network; this allowed to isolate 600 papers, which were manually examined and annotated for enriched human gene ontology according to BiNGO v.3.5.0.

\section{Cells}

African green monkey kidney Vero E6 cell line was obtained from American Type Culture Collection (ATCC, Manassas, VA, USA) and maintained in Dulbecco's Modified Eagle Medium (DMEM; Gibco, Thermo-Fisher, Waltham, MA, USA) supplemented with $10 \%$ fetal bovine serum (FBS; Gibco, Thermo-Fisher) at $37^{\circ} \mathrm{C}$ in a humidified atmosphere of $5 \% \mathrm{CO}_{2}$.

Calu-3 (human, Caucasian, lung, adenocarcinoma) cell line was obtained from ATCC and maintained in Minimum Essential Medium (MEM; Gibco, Thermo-Fisher) supplemented with $10 \%$ fetal bovine serum (FBS; Gibco, Thermo-Fisher) at $37^{\circ} \mathrm{C}$ in a humidified atmosphere of $5 \% \mathrm{CO}_{2}$.

\section{Virus}

Different SARS-CoV-2 variants isolated from COVID-19 patients' respiratory samples were used. The identity of each variant was verified by metagenomic sequencing. Genomic data of SARS-CoV2, belonging to the B.1 lineage, are available at EBI (under study accession number: PRJEB38101) $[67,68]$.

Below the list of the viral strains used to assess the activity of raloxifene against viral variants:

- Human 2019-nCoV strain 2019-nCoV/Italy-INMI1, clade V (Ref-SKU: 008V-03893, EVAg portal), and isolated in January 2020 from a chinese patient (control infection) (named Wuhan)

- SARS-CoV-2 isolate SARS-CoV-2/Human/ITA/PAVIA10734/2020, clade G, D614G (S) (Ref-SKU: 008V-04005, EVAg portal), named D614G, isolated in Lombardy in February 2020 
- SARS-CoV-2 isolate hCoV-19/Italy/LAZ-INMI-82isl/2020, clade GV, A222V, D614G (S) (Ref-SKU: 008V-04048, EVAg portal), named GV and representig the dominant strain circulating in Europe from April to December 2020.

- SARS-CoV-2 variant VOC 202012/01, isolate hCoV-19/Italy/CAM-INMI-118isl/2020, clade GR, $\Delta 69-70, \Delta 144$, N501Y, A570D, D614G, P681H, T716I (S) (Ref-SKU: 008V-04050, EVAg portal), named VOC B.1.1.7 and representing the variant of major concern from UK

- SARS-CoV-2 variant GR/501Y.V3, isolate hCoV-19/Italy/LAZ-INMI-216isl/2021, clade GR, PANGO lineage P.1, K417T, E484K, N501Y (S) (Ref-SKU: 008V-04101, EVAg portal), named VOC P1 representing the variant of major concern from Brazil

- SARS-CoV-2 variant VOC SA/B.1.351, obtained by GHSAG (Public Health England), named B.1.351 and representing the variant of major concern from South Africa

- SARS-CoV-2 variant VOC G Delta/B1.617.2 isolate hCoV-19/Italy/LAZ-INMI-648/2021 (EPI_ISL_2000624), named VOC B.1.617.2 representing the variant of major concern from India

All the infection experiments were performed in a biosafety level-3 (BLS-3) laboratory at a multiplicity of infection (MOI) of 0.05 .

\section{Cell viability studies of raloxifene}

Cells were seeded into 24-well plates $\left(2.5 \times 10^{4}\right.$ cells/well) in DMEM supplemented with 10\% FBS, and treated with different doses of raloxifene $(1.25,2.5,5,10,15,20,25$ and $30 \mu \mathrm{M})$ at $37^{\circ} \mathrm{C}$ for 48 h. Cell viability was estimated by measuring the ATP levels using CellTiter-Glo (Promega, Madison, WI, USA).

\section{Evaluation of antiviral efficacy of raloxifene}

Cells were infected at $37^{\circ} \mathrm{C}$ for $1 \mathrm{~h}$ with the SARS-CoV-2 isolate at a MOI of 0.05 . Infection was carryed out in DMEM without FBS. Then, the virus was removed and cells washed with warm phosphate buffered saline (PBS) and cultured with medium containing 2\% FBS in the presence or in the absence of raloxifene. The compound was used at the concentration of 1.25, 2.5, 5, 10 and 15 $\mu \mathrm{M}$ and both cells and supernatants were collected for further analysis $48 \mathrm{~h}$ post infection (p.i). 


\section{Plaque Assay}

Cells were seeded at a density of $5 \times 10^{5}$ cells/well in a 12 -well plate and incubated at $37^{\circ} \mathrm{C}$ for $24 \mathrm{~h}$. Supernatants from infected cells were serially diluted in DMEM without FBS and added to the cells. After $1 \mathrm{~h}$ incubation, media were removed and cells washed with warm PBS. Then cells were covered with an overlay consisting of DMEM with $0.4 \%$ SeaPlaque (Lonza, Basel, Switzerland). The plates were further incubated at $37^{\circ} \mathrm{C}$ for $48 \mathrm{~h}$. Cells were fixed with $10 \%$ formaldehyde at room temperature for $3 \mathrm{~h}$. Formaldehyde was aspirated and the agarose overlay was removed. Cells were then stained with crystal violet ( $1 \% \mathrm{CV}$ w/v in a $20 \%$ ethanol solution), and viral titer (PFU/mL) of SARS-CoV2 was determined by counting the number of plaques.

\section{Viral RNA extraction and quantitative real-time RT-PCR (qRT-PCR)}

RNA was extracted from clarified cell culture supernatants $(16,000 \mathrm{~g}$ x $10 \mathrm{~min})$ and from infected cells using QIAamp Viral RNA Mini Kit and RNeasy Plus mini kit (Qiagen, Hilden, Germany), respectively, according to the manufacturer's instructions.

RNA was eluted in $30 \mu 1$ of RNase-free water and stored at $-80^{\circ} \mathrm{C}$ till use. The qRT-PCR was carriedout following previously described procedures with minor modifications [69]. Briefly, reverse transcription and amplification of the $\mathrm{S}$ gene were performed using the one-step QuantiFast Sybr Green RT-PCR mix (Qiagen) as follows: $50^{\circ} \mathrm{C}$ for $10 \mathrm{~min}, 95^{\circ} \mathrm{C}$ for $5 \mathrm{~min}$; $95^{\circ} \mathrm{C}$ for $10 \mathrm{sec}, 60^{\circ} \mathrm{C}$ for $30 \mathrm{sec}$ (40 cycles) (primers: RBD-qF1: 5'-CAATGGTTTAACAGGCACAGG-3' and RBD-qR1: 5'-CTCAAGTGTCTGTGGATCACG-3). A standard curve was generated by determination of copy numbers derived from serial dilutions $\left(10^{3}-10^{9}\right.$ copies) of a pGEM T-easy vector (Promega, Madison, WI, USA) containing the receptor binding domain of the $\mathrm{S}$ gene (primers: RBD-F: 5'GCTGGATCCCCTAATATTACAAACTTGTGCC-3'; RBD-R: 5 TGCCTCGAGCTCAAGTGTCTGTGGATCAC-3').

\section{Western blot analysis}

Western blot was carried-out following previously described procedures with minor modifications [70]. Protein samples $(30 \mu \mathrm{g})$ obtained from lysis in RIPA buffer (Cell Signaling Technology, Danvers, MA, USA) of infected cells were separated on 10\% SDS-PAGE and then transferred onto 
polyvinylidene difluoride (PVDF) membranes (Millipore, Sigma, Burlington, MA, USA). After being blocked with $3 \%$ BSA in TBS buffer containing $0.05 \%$ Tween 20 , the blot was probed with a human serum (1:1000 dilution) containing IgG to the SARS-CoV-2 nucleoprotein (NP) and with mouse anti-human GAPDH monoclonal antibody (G-9: Santa Cruz Biotechnology, Dallas, TX, USA). The antigen-antibody complexes were detected using peroxidase-conjugated goat anti-human or goat anti-mouse IgG (Sigma) and revealed using the enhanced chemiluminescence (ECL) system (Santa Cruz Biotechnology).

\section{Evaluation of antiviral efficacy of raloxifene on SARS-CoV2 variants}

Vero E6 cells were infected at $37^{\circ} \mathrm{C}$ for $1 \mathrm{~h}$ with the SARS-CoV-2 strains indicated in the Virus section at a MOI of 0.05 in 96 well plates. Infection was carryed out in MEM (Sigma) without FBS (Gibco). Then, the virus was removed and cells washed with warm phosphate buffered saline (PBS) and cultured with medium containing $2 \%$ FBS in the presence or absence of raloxifene at different doses $(0.23,0.47,0.94,1.88,3.75,7.5,15 \mu \mathrm{M})$ at $37^{\circ} \mathrm{C}$ and $5 \% \mathrm{CO} 2$ up to $72 \mathrm{~h}$. To determine antiviral efficacy of raloxifene, cell viability and viral induced CPE were mesured in not infected and infected cells treated with serial dilution of the drug, staining the cells with a solution of Crystal Violet (Diapath) and 2\% formaldehyde. After $30 \mathrm{~min}$, the fixing solution was removed by washing with tap water, and cell viability was measured by a photometer at $595 \mathrm{~nm}$ (Synergy ${ }^{\mathrm{TM}}$ HTX Multi-Mode Microplate Reader, Biotek, Winooski, VT, USA).

The percentage of viable cells for each condition was calculated compared to infected-not-treated (set as $0 \%$ ) and not-infected-not-treated cells (set as $100 \%$ ). The effect of raloxifene on cell viability was also checked by crystal violet/ $2 \%$ formaldheyde staining in each experiment performed for SARSCoV-2 variants study.

\section{Data analysis}

The half-cytotoxic concentration $\left(\mathrm{CC}_{50}\right)$ and the half-maximal inhibitory concentration ( $\left.\mathrm{IC}_{50}\right)$ for raloxifene were calculated from concentration-effect-curves after non-linear regression analysis using GraphPad Prism8. The selectivity index (SI) for raloxifene was calculated as the ratio of $\mathrm{CC}_{50}$ over $\mathrm{IC}_{50}$ [71]. 


\section{Statistical analysis}

Data for the in vitro experiments performed were analyzed for statistical significance using the 1-way ANOVA, and the Bonferroni post-test was used to compare data. Differences were considered significant when $p<0.05$. Statistical tests were performed using GraphPad Prism 8.

\section{Results}

\section{In vitro effects of raloxifene on metabolism and SARS-CoV-2 infection in different cell lines}

At first, a standard assay was carried out to measure the activity of raloxifene on cell metabolism. To this end, Vero E6 cells were cultured for $48 \mathrm{~h}$ in the absence or presence of different drug concentrations (range from $1.25 \mu \mathrm{M}$ to $30 \mu \mathrm{M}$ ). As shown in Figure 1A, raloxifene-treated Vero E6 cells showed a normal surface-adherent phenotype until the concentration of $15 \mu \mathrm{M}$. A drugdependent cytopathic effect was evident at concentration of $20 \mu \mathrm{M}$, involving the entire monolayer at a concentration of $25 \mu \mathrm{M}$ and $30 \mu \mathrm{M}$. At the same time, raloxifene shows a slight effect on the extent of cellular ATP accumulation at a concentration ranging from $1.25 \mu \mathrm{M}$ to $15 \mu \mathrm{M}(87 \%$ and $70 \%$, respectively). At higher doses, raloxifene showed a dose-dependent effect on ATP accumulation, reaching 56\%, 35\% and $0.6 \%$ at $20 \mu \mathrm{M}, 25 \mu \mathrm{M}$ and $30 \mu \mathrm{M}$ (Figure 1B). The $\mathrm{CC}_{50}$ of raloxifene in Vero E6 cells was determined to be $18.4 \mu \mathrm{M}$.

Next, to assess the antiviral activity of raloxifene, Vero E6 cells were infected with SARS-CoV-2 (B.1 lineage) at a MOI of 0.05 [67]. Specifically, Vero E6 were infected with SARS-CoV-2, and $1 \mathrm{~h}$ later cultured in the absence or presence of different raloxifene concentrations (range from $1.25 \mu \mathrm{M}$ to $15 \mu \mathrm{M})$. Raloxifene efficiently inhibits viral replication. In particular, viral genome copy numbers evaluated on supernatants collected at $48 \mathrm{~h}$ p.i. by qRT-PCR, showed a significant reduction of the virus yield already at $2.5 \mu \mathrm{M}$ raloxifene concentration (2.9-fold reduction), with a maximal reduction at $15 \mu \mathrm{M}$ (1400-fold reduction) (Figure 1C). Raloxifene also displayed a dose-dependent inhibition of viral replication in Vero E6 cells, as determined by infectious viral titers, exhibiting a 70\% reduction of viral titer at a concentration of $5 \mu \mathrm{M}$, with $94 \%$ to $100 \%$ inhibition at $10 \mu \mathrm{M}$ and $15 \mu \mathrm{M}$, respectively (Figure 1D). Raloxifene efficacy was then confirmed at intracellular level. Quantification of viral RNA in SARS-CoV-2-infected cells showed a significant reduction of intracellular SARSCoV-2 genome copy number already at $10 \mu \mathrm{M}$ and a 99-fold reduction at $15 \mu \mathrm{M}$ (Figure 1E). 
Accordingly, western blot (WB) analysis showed a dose-dependent inhibition of SARS-CoV-2 upon raloxifene treatment with $43 \%$ reduction of NP viral protein expression at a concentration of $5 \mu \mathrm{M}$, with $65 \%$ and $97 \%$ reduction at $10 \mu \mathrm{M}$ and $15 \mu \mathrm{M}$, respectively (Figure $1 \mathrm{~F}$ ). Raloxifene $\mathrm{IC}_{50}$ value was calculated to be $3.3 \mu \mathrm{M}$. SI was then calculated and found to be 5.6. We then tested the raloxifene activity on Calu-3 cells as a model of human pulmonary cell line. The $\mathrm{CC}_{50}$ value was determined, as above, and found to be $24.4 \mu \mathrm{M}$ (Figure 2A). Next, Calu-3 cells were infected as described above. Supernatants were collected at 48 h p.i., and tested for viral genome copy numbers by qRT-PCR. As shown in Figure 2B, the treatment significantly reduced the virus yield. In particular, raloxifene displayed a dose-dependent inhibition of viral replication, as determined by infectious viral titers, exhibiting a $67 \%$ reduction of viral titer at a concentration as low as $10 \mu \mathrm{M}$, with $96 \%$ and $98 \%$

A



B



E

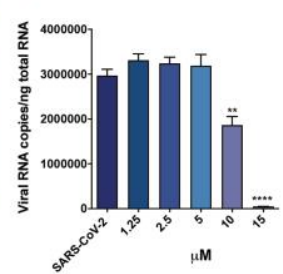

C

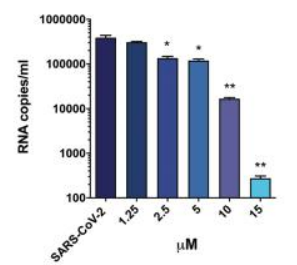

F

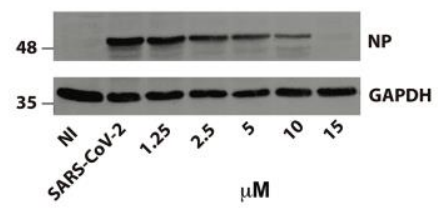

D
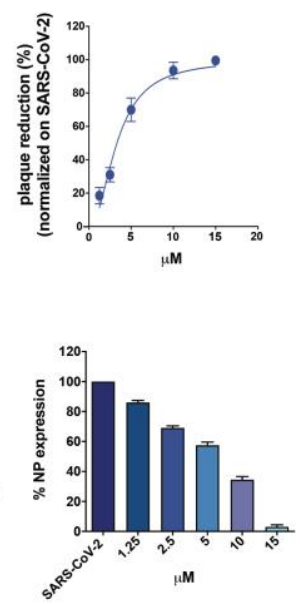

Figure 1. Effect of Raloxifene on Vero E6 cells. Vero E6 cells were cultured for $48 \mathrm{~h}$ in the absence or in the presence of raloxifene at different concentrations. (A) 10× bright-field images of Vero E6 cells after incubation for $48 \mathrm{~h}$ at $37^{\circ} \mathrm{C}$ with the indicated raloxifene concentrations. (B) CellTiter-Glo was used to measure the antimetabolic effect of raloxifene. (C-F) Vero E6 cells were infected with SARS-CoV-2 and cultured in the absence or in the presence of different doses of raloxifene. (C) Viral yield in cell supernatants was quantitated by qRT-PCR. (D) Viral titer in cell supernatants was evaluated by plaque assay and plotted as percentage of plaque reduction compared to SARS-CoV-2. (E) Quantitation of SARS-CoV-2 genomes at the intracellular level by qRT-PCR. (F) NP expression in infected cells was analyzed by western blot (left panel). Densitometric analysis of western blot is shown in the right panel. Graph represents the percentage of NP expression. Data are representative of two independent experiments with similar results. All the experiments were 

\pm standard error of the mean *, $\mathrm{P}<0.05 ; * *, \mathrm{P}<0.01 ; * * *, \mathrm{P}<0.0001$.

A

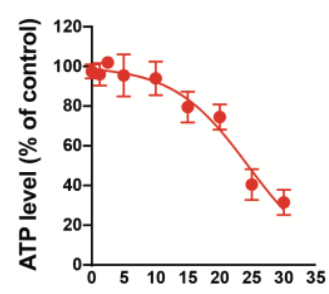

D

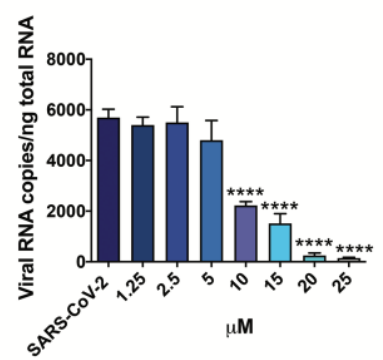

B

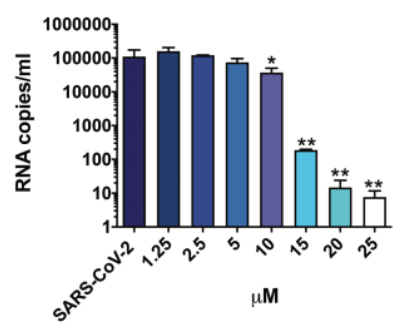

$\mathbf{E}$

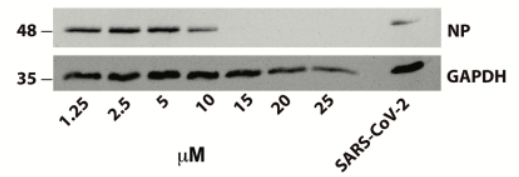

c
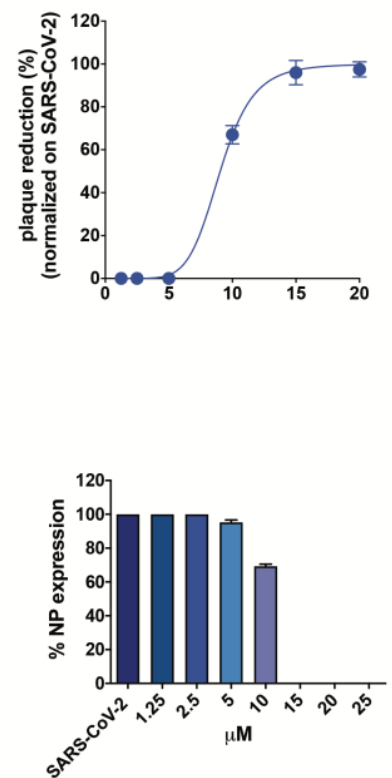

Figure 2. Effect of Raloxifene on Calu-3 cells. (A) Calu-3 cells were cultured for $48 \mathrm{~h}$ in absence or in the presence of raloxifene at different concentrations. CellTiter-Glo was used to measure, the antimetabolic effect of raloxifene. (B-E) Cells were infected with SARS-CoV-2 and cultured in the absence or in the presence of different doses of raloxifene. (B) Viral yield in cell supernatants was quantitated by qRT-PCR. (C) Viral titer in cell supernatants was evaluated by plaque assay and plotted as percentage of plaque reduction compared to SARS-CoV-2. (D) Quantitation of SARS-CoV-2 genomes at the intracellular level by qRT-PCR. (E) Nucleocapsid (NP) protein expression in infected cells was analyzed by western blot (left panel). Densitometric analyses of western blot results are shown. Graph represents the percentage of NP protein expression. Data are representative of two independent experiments with similar results. All the experiments were performed at least in three independent replicates and pictures shown are representative. Data are presented as the mean + standard error of the mean *, $\mathrm{P}<0.05 ; * *, \mathrm{P}<0.01$; ****, $\mathrm{P}<0.0001$.

\section{Raloxifene exerts antiviral activity on SARS-CoV-2 variants}

We then performed a systematic study of the antiviral efficacy of raloxifene on the most common variants on Vero E6 cells (Figure 3). Different viral strains were used: the wild type isolated in January 2020 from Chinese patient (named Wuhan), two different isolates for the D614G spike 
GV and D614G) and the variants of major concern originated in UK, Brazil, South Africa and India (named VOC B1.1.7, VOC P.1, VOC B1.351, and VOC B1.617.2, respectively). We first determined the time-window in which CPE appeared for each variant. The CPE was evident at $48 \mathrm{~h}$ for all the tested strains but VOC B1.1.7 and VOC P.1 variant, for which evident CPE appeared later (56h and $72 \mathrm{~h}$, respectively). In parallel, uninfected cells were cultured in the presence of different doses of raloxifene to evaluate possible cytotoxicity due to the treatment. In cells treated with drug at $15 \mu \mathrm{M}$, we observed a reduced percentage of viable cells as revealed by crystal violet staining $(82.9+/-8.69 \%$ and $76.3+/-5.84 \%$, at 48 and $72 \mathrm{~h}$ respectively, compared to $100 \%$ in untreated cells). No significant effect on cell viability with lower dug concentrations $(7.5$ to $0.23 \mu \mathrm{M})$ was observed. To determine antiviral efficacy of the drug, CPE was measured in infected cells treated with seven two-fold serial dilutions of raloxifene ( 15 to $0.23 \mu \mathrm{M})$ using the time windows identified for each strain. The drug was able to recover cell viability in Vero E6 cells infected with all the tested viral strains. The halfmaximal inhibitory concentration $\left(\mathrm{IC}_{50}\right.$ ) calculated on recovering of cell viability varied from 4.50 to $7.99 \mu \mathrm{M}$ depending on the strain (Figure 3), showing a strong antiviral activity against all the variants under investigation.



\begin{tabular}{|rcc}
\hline & IC50 & 95\% CI \\
\hline Wuhan & 5,92 & $5,29-6,54$ \\
D614G & 7,48 & $6,38-8,90$ \\
\hline GV & 4,51 & $3,22-6,47$ \\
\hline VOCB.1.1.7 & 6,65 & $5,25-8,54$ \\
\hline VOCP.1 & 5,70 & $4,61-7,22$ \\
\hline VOC B.1.351 & 4,58 & $3,81-5,55$ \\
\hline VOC B.1.617.2 & 7,99 & $5,91-11,68$ \\
\hline
\end{tabular}
graph shows the inhibition of CPE observed at different concentration of raloxifene. The IC50 calculated by non linear regression are shown in the table. Percentage of viable cells calculated on not treated not infected $=100 \%$; not treated SARS-CoV-2 infected cells $=0 \%$. Bars indicate SD. 


\section{System Biology screening to investigate poly-pharmacological effects of raloxifene against SARS-}

\section{CoV-2 infection}

Besides being able to directly bind viral proteins such as Spike, raloxifene may exert a range of other modulatory effects on the infection by SARS-CoV-2. In a recent paper we reviewed the possible links between ER modulation and host response to viral infections against different viruses, suggesting a therapeutic potential for SERMs in the control of COVID-19 infection [38]. Aiming to strengthen the above hypothesis we built a molecular network connecting the human-virus interactome and those proteins known to be involved in the COVID-19 pathogenesis, as described in the Materials \& methods section. The resulting network was in turn used to generate a list of proteins; each member of this list was used as a probe to screen all the papers on raloxifene nominating the proteins relevant for the SARS-CoV-2 infection. In this way, three functional groups of human genes involved in the biology of the viral infection and potentially modulated by raloxifene were identified:

1. A group of genes including those modulated by the raloxifene molecular target, specifically ESR2, and connected to inflammation;

2. A group of genes including those expressed in the lungs which are modulated by the raloxifene molecular target, specifically ESR; when deregulated, the corresponding genes cause severe asthma, in agreement with the enrichment in this group of genes whose unfavorable variants cause worse respiratory consequences, according to GWAS studies;

3. A group of genes directly modulated by the virus, both during the cell entry phase and the replication phase, which also include proteins upstream or downstream of some raloxifenecontrolled pathways.

Taken all together, these results suggest a potential poly-pharmacological effect of raloxifene in COVID-19 as anti-inflammatory, respiratory and antiviral.

Effect on inflammation: Looking at the genes identified as linked to the promotion of inflammation by the virus, it is relevant to keep in mind that one of the clinically validated targets of the anti COVID-19 therapy is the cytokine Interleukin-6 (IL-6) [72]. A downregulation of the inflammatory signal and of the IL-6 expression was found with raloxifene in a clinical setting [73]. Besides IL-6, other serum cytokines (i.e. TNF-alpha and TGF-beta1) involved in the cytokines storm due to SARS$\mathrm{CoV}-2$ are regulated by raloxifene.

Effect on respiratory response: Genes regulating the production of nitric oxide are implicated in the vascular and respiratory response to the viral infection. Treatment of rats with raloxifene was shown to upregulate the expression of eNOS (NOS3) in rat thoracic aorta, after complexing with the 
ESR $_{2}$ expressed in lungs. This is expected to exert a potentially important vasculo-protective effect, and eventually to contribute to clinical improvements in ARDS and pulmonary hypertension [74]. Other compounds, e.g. Rho kinase (ROK) inhibitors, phosphodiesterase-5 inhibitors [75], omentin [76] were also shown to activate the eNOS (NOS3) pathway, with protective effect for ARDS and related inflammation in experimental models. In ARDS patients, the effects of inhaled nitric oxide on the reduction of pulmonary blood pressure and on the improvement of oxygenation, offered the rationale for a clinical trial in severe COVID-19 patients (NCT04388683).

Effect on antiviral action: Consistently with the identification of the genes from the GWAS study, a direct antiviral action of raloxifene, in terms of inhibition of viral replication and/or infection, was found in several different contexts like in vitro against EbolaVirus [58, 59, 77], against HBV [62], and against $\mathrm{HCV}$ [60]; in human female cells from nasal epithelium against the Influenza Virus A [64], and also in a randomized clinical trial on 123 postmenopausal women, against HCV [65].

\section{Discussion}

Raloxifene, a second generation SERM, was previously proposed as a potential candidate for the treatment of COVID-19 patients due to the in silico predicted possibility to interfere with the viral replication and disease progression with multiple mechanisms of action both ER dependent and independent [38].

In this paper we report for the first time the in vitro characterization of the antiviral activity of raloxifene against SARS-CoV-2 infection using two relevant experimental systems, Vero E6 monkey kidney cells and human pulmonary Calu-3 cells. SARS-CoV-2 infected monkey Vero E6 cells are commonly used to study coronavirus infection as they support viral replication to high titres and highly express ACE-2 receptor [78-82] that plays an essential role for SARS-CoV-2 entry into the cells [83]. SARS-CoV-2 infected human Calu-3 cells are a relevant and predictive model because of airway epithelial origin [84]. In both assays the results confirmed that raloxifene blocks with high efficiency SARS-CoV-2 replication.

The characterization was completed testing raloxifene also against all the most common circulating SARS-CoV-2 variants of clinical relevance, confirming that it maintains a high and consistent activity, thus reinforcing the interest on its potential clinical use as antiviral agent in COVID-19 patients. 
Raloxifene cytotoxicity was assessed with two independent assays: the first measuring the activity of the compound on cell replication, the second on cellular metabolism. With both approaches we found that the CC50 was attested at high micromolar range, which is far from the low micromolar range in which the antiviral activity was observed. The selectivity index (SI) of the drug was superimposable in the two experimental models in the range of 2 to 7 . In general, the value of SI for a drug with direct antiviral activity is greater than 1; the higher the SI value, the more effective and safer the drug is. Some authors [85-87] report a limit value of SI $=4$ to define a compound as a good compound with direct antiviral activity. The SI value found in the models is therefore indicative of a molecule with a significant antiviral activity and with an activity/toxicity profile consistent with a possible translation to human clinical trials. In addition, raloxifene is a drug that has been used for a long time, and its safety profile is supported by a huge volume of clinical data from long term treatments [88-90]. The occurrence of thromboembolic events, even though rare, in patients treated with raloxifene has to be regarded with particular caution due to the high risk of thromboembolic manifestations in COVID patients. A short duration of treatment and the careful avoidance to treat patients with concomitant risks of thromboembolic events are recommended.

Among SERMs raloxifene has a unique risk/benefit profile built on a large safety database not limited to oncological patients, like for other SERMs, but on a large use in postmenopausal women for the management of osteoporosis, including men treated for a variety of indications. The potential of SERMs, and in particular of raloxifene, found a promising confirmation in a recent retrospective study on a large population of cancer patients that demonstrated a protective effect on SARS-CoV-2 infection and a significant reduction of severity and duration in the subpopulation of patients treated with raloxifene [91].

A system biology study was also conducted with the aim to match the available information on gene and pathways regulated by raloxifene against a Cytoskape-generated human SARS-CoV-2 interactome network. The results of the study strongly support the concept that raloxifene may positively influence the course of SARS-COV-2 infection by modulating three functional groups of human genes, all of them playing a key role in the biology of the viral infection. The systematic data analysis went further, confirming the putative antiviral activity, and also highlighting the potential of raloxifene to exert both an antinflammatory action by downregulating the expression of key mediators of the cytokine storm, and a vasculo-protective effect by upregulating eNOS expression (NOS3) [74]. These findings on one hand are in agreement with previous papers highlighting the protective effect of estrogen signalling in the context of COVID-19 infection, and on the other hand confirm the 
specific characteristics of raloxifene as an ideal candidate to put to a test the hypothesis in the clinics due to ist peculiar mechanism within the class of SERMs and its potential ability to exert a pleiotropic effect by targeting viral and host targets with a key role in the disease progression and exacerbation.

\section{References}

1. Cui, J., F. Li, and Z.L. Shi, Origin and evolution of pathogenic coronaviruses. Nat Rev Microbiol, 2019. 17(3): p. 181-192.

2. Su, S., et al., Epidemiology, Genetic Recombination, and Pathogenesis of Coronaviruses. Trends Microbiol, 2016. 24(6): p. 490-502.

3. Hu, B., et al., Characteristics of SARS-CoV-2 and COVID-19. Nat Rev Microbiol, 2021. 19(3): p. 141154.

4. Raoult, D., et al., Coronavirus infections: Epidemiological, clinical and immunological features and hypotheses. Cell Stress, 2020. 4(4): p. 66-75.

5. Lu, R., et al., Genomic characterisation and epidemiology of 2019 novel coronavirus: implications for virus origins and receptor binding. Lancet, 2020. 395(10224): p. 565-574.

6. Huang, C., et al., Clinical features of patients infected with 2019 novel coronavirus in Wuhan, China. Lancet, 2020. 395(10223): p. 497-506.

7. Wang, D., et al., Clinical Characteristics of 138 Hospitalized Patients With 2019 Novel CoronavirusInfected Pneumonia in Wuhan, China. JAMA, 2020. 323(11): p. 1061-1069.

8. Torjesen, I., Covid-19 will become endemic but with decreased potency over time, scientists believe. BMJ, 2021. 372: p. n494.

9. Veldhoen, M. and J.P. Simas, Endemic SARS-CoV-2 will maintain post-pandemic immunity. Nat Rev Immunol, 2021. 21(3): p. 131-132.

10. Isabel, S., et al., Evolutionary and structural analyses of SARS-CoV-2 D614G spike protein mutation now documented worldwide. Sci Rep, 2020. 10(1): p. 14031.

11. Koyama, T., D. Platt, and L. Parida, Variant analysis of SARS-CoV-2 genomes. Bull World Health Organ, 2020. 98(7): p. 495-504.

12. Korber, B., et al., Tracking Changes in SARS-CoV-2 Spike: Evidence that D614G Increases Infectivity of the COVID-19 Virus. Cell, 2020. 182(4): p. 812-827 e19.

13. Mascola, J.R., B.S. Graham, and A.S. Fauci, SARS-CoV-2 Viral Variants-Tackling a Moving Target. JAMA, 2021. 325(13): p. 1261-1262.

14. Funk, T., et al., Characteristics of SARS-CoV-2 variants of concern B.1.1.7, B.1.351 or P.1: data from seven EU/EEA countries, weeks 38/2020 to 10/2021. Euro Surveill, 2021. 26(16).

15. Davies, N.G., et al., Estimated transmissibility and impact of SARS-CoV-2 lineage B.1.1.7 in England. Science, 2021. 372(6538).

16. Moyo-Gwete, T., et al., SARS-CoV-2 501Y.V2 (B.1.351) elicits cross-reactive neutralizing antibodies. bioRxiv, 2021.

17. Tegally, H., et al., Detection of a SARS-CoV-2 variant of concern in South Africa. Nature, 2021. 592(7854): p. 438-443.

18. Faria, N.R., et al., Genomics and epidemiology of the P.1 SARS-CoV-2 lineage in Manaus, Brazil. Science, 2021. 372(6544): p. 815-821.

19. Sabino, E.C., et al., Resurgence of COVID-19 in Manaus, Brazil, despite high seroprevalence. Lancet, 2021. 397(10273): p. 452-455.

20. Deng, X., et al., Transmission, infectivity, and antibody neutralization of an emerging SARS-CoV-2 variant in California carrying a L452R spike protein mutation. medRxiv, 2021. 
21. Aleem, A., A.B. Akbar Samad, and A.K. Slenker, Emerging Variants of SARS-CoV-2 And Novel Therapeutics Against Coronavirus (COVID-19), in StatPearls. 2021: Treasure Island (FL).

22. Zhang, Q., et al., Molecular mechanism of interaction between SARS-CoV-2 and host cells and interventional therapy. Signal Transduct Target Ther, 2021. 6(1): p. 233.

23. Wang, Q., et al., Structural and Functional Basis of SARS-CoV-2 Entry by Using Human ACE2. Cell, 2020. 181(4): p. 894-904 e9.

24. Hoffmann, M., et al., SARS-CoV-2 Cell Entry Depends on ACE2 and TMPRSS2 and Is Blocked by a Clinically Proven Protease Inhibitor. Cell, 2020. 181(2): p. 271-280 e8.

25. Daly, J.L., et al., Neuropilin-1 is a host factor for SARS-CoV-2 infection. Science, 2020. 370(6518): p. 861-865.

26. Cantuti-Castelvetri, L., et al., Neuropilin-1 facilitates SARS-CoV-2 cell entry and infectivity. Science, 2020. 370(6518): p. 856-860.

27. Qiao, Y., et al., Targeting transcriptional regulation of SARS-CoV-2 entry factors ACE2 and TMPRSS2. Proc Natl Acad Sci U S A, 2020.

28. Bravaccini, S., et al., Estrogen and Androgen Receptor Inhibitors: Unexpected Allies in the Fight Against COVID-19. Cell Transplant, 2021. 30: p. 963689721991477.

29. Strope, J.D., C.H. Chau, and W.D. Figg, Are sex discordant outcomes in COVID-19 related to sex hormones? Semin Oncol, 2020. 47(5): p. 335-340.

30. Khan, D. and S. Ansar Ahmed, The Immune System Is a Natural Target for Estrogen Action: Opposing Effects of Estrogen in Two Prototypical Autoimmune Diseases. Front Immunol, 2015. 6: p. 635.

31. Straub, R.H., The complex role of estrogens in inflammation. Endocr Rev, 2007. 28(5): p. 521-74.

32. Kovats, S., Estrogen receptors regulate innate immune cells and signaling pathways. Cell Immunol, 2015. 294(2): p. 63-9.

33. Stilhano, R.S., et al., SARS-CoV-2 and the possible connection to ERs, ACE2, and RAGE: Focus on susceptibility factors. FASEB J, 2020. 34(11): p. 14103-14119.

34. Mauvais-Jarvis, F., S.L. Klein, and E.R. Levin, Estradiol, Progesterone, Immunomodulation, and COVID-19 Outcomes. Endocrinology, 2020. 161(9).

35. Haitao, T., et al., COVID-19 and Sex Differences: Mechanisms and Biomarkers. Mayo Clin Proc, 2020. 95(10): p. 2189-2203.

36. Abate, B.B., et al., Sex difference in coronavirus disease (COVID-19): a systematic review and metaanalysis. BMJ Open, 2020. 10(10): p. e040129.

37. Lipsa, A. and J.S. Prabhu, Gender disparity in COVID-19: Role of sex steroid hormones. Asian Pac J Trop Med, 2021. 14(1): p. 5-9.

38. Allegretti, M., et al., Repurposing the estrogen receptor modulator raloxifene to treat SARS-CoV-2 infection. Cell Death Differ, 2021.

39. Gervasoni, S., et al., A Comprehensive Mapping of the Druggable Cavities within the SARS-CoV-2 Therapeutically Relevant Proteins by Combining Pocket and Docking Searches as Implemented in Pockets 2.0. Int J Mol Sci, 2020. 21(14).

40. Grottesi, A., et al., Computational Studies of SARS-CoV-2 3CLpro: Insights from MD Simulations. Int J Mol Sci, 2020. 21(15).

41. Manelfi, C., et al., Combining Different Docking Engines and Consensus Strategies to Design and Validate Optimized Virtual Screening Protocols for the SARS-CoV-2 3CL Protease. Molecules, 2021. 26(4).

42. Gossen, J., et al., A Blueprint for High Affinity SARS-CoV-2 Mpro Inhibitors from Activity-Based Compound Library Screening Guided by Analysis of Protein Dynamics. ACS Pharmacol Transl Sci, 2021. 4(3): p. 1079-1095.

43. Muchmore, D.B., Raloxifene: A selective estrogen receptor modulator (SERM) with multiple target system effects. Oncologist, 2000. 5(5): p. 388-92.

44. Goldstein, S.R., Selective estrogen receptor modulators and bone health. Climacteric, 2021: p. 1-4.

45. Hernandez, E., et al., Effects of raloxifene on bone metabolism and serum lipids in postmenopausal women on chronic hemodialysis. Kidney Int, 2003. 63(6): p. 2269-74. 
46. Lewis, J.S. and V.C. Jordan, Selective estrogen receptor modulators (SERMs): mechanisms of anticarcinogenesis and drug resistance. Mutat Res, 2005. 591(1-2): p. 247-63.

47. Walsh, B.W., et al., Effects of raloxifene on serum lipids and coagulation factors in healthy postmenopausal women. JAMA, 1998. 279(18): p. 1445-51.

48. Messalli, E.M. and C. Scaffa, Long-term safety and efficacy of raloxifene in the prevention and treatment of postmenopausal osteoporosis: an update. Int J Womens Health, 2010. 1: p. 11-20.

49. Grady, D., et al., Safety and adverse effects associated with raloxifene: multiple outcomes of raloxifene evaluation. Obstet Gynecol, 2004. 104(4): p. 837-44.

50. Levenson, A.S., et al., Understanding the Antiestrogenic Actions of Raloxifene and a Mechanism of Drug Resistance to Tamoxifen. Breast Cancer, 1998. 5(2): p. 99-106.

51. MacGregor, J.I. and V.C. Jordan, Basic guide to the mechanisms of antiestrogen action. Pharmacol Rev, 1998. 50(2): p. 151-96.

52. Clemett, D. and C.M. Spencer, Raloxifene: a review of its use in postmenopausal osteoporosis. Drugs, 2000. 60(2): p. 379-411.

53. Vogel, V.G., et al., Effects of tamoxifen vs raloxifene on the risk of developing invasive breast cancer and other disease outcomes: the NSABP Study of Tamoxifen and Raloxifene (STAR) P-2 trial. JAMA, 2006. 295(23): p. 2727-41.

54. DeMichele, A., et al., Impact of raloxifene or tamoxifen use on endometrial cancer risk: a populationbased case-control study. J Clin Oncol, 2008. 26(25): p. 4151-9.

55. Weickert, T.W., et al., Adjunctive raloxifene treatment improves attention and memory in men and women with schizophrenia. Mol Psychiatry, 2015. 20(6): p. 685-94.

56. Smith, M.R., et al., Raloxifene to prevent gonadotropin-releasing hormone agonist-induced bone loss in men with prostate cancer: a randomized controlled trial. J Clin Endocrinol Metab, 2004. 89(8): p. 3841-6.

57. Doran, P.M., et al., Effects of raloxifene, a selective estrogen receptor modulator, on bone turnover markers and serum sex steroid and lipid levels in elderly men. J Bone Miner Res, 2001. 16(11): p. 2118-25.

58. Montoya, M.C. and D.J. Krysan, Repurposing Estrogen Receptor Antagonists for the Treatment of Infectious Disease. mBio, 2018. 9(6).

59. Yoon, Y.S., et al., Antiviral activity of sertindole, raloxifene and ibutamoren against transcription and replication-competent Ebola virus-like particles. BMB Rep, 2020. 53(3): p. 166-171.

60. Takeda, M., et al., Raloxifene inhibits hepatitis $C$ virus infection and replication. FEBS Open Bio, 2012. 2: p. 279-83.

61. Murakami, Y., et al., Selective estrogen receptor modulators inhibit hepatitis $C$ virus infection at multiple steps of the virus life cycle. Microbes Infect, 2013. 15(1): p. 45-55.

62. Lamontagne, J., et al., Screening and identification of compounds with antiviral activity against hepatitis $B$ virus using a safe compound library and novel real-time immune-absorbance PCR-based high throughput system. Antiviral Res, 2013. 98(1): p. 19-26.

63. Eyre, N.S., et al., Identification of Estrogen Receptor Modulators as Inhibitors of Flavivirus Infection. Antimicrob Agents Chemother, 2020. 64(8).

64. Peretz, J., et al., Estrogenic compounds reduce influenza A virus replication in primary human nasal epithelial cells derived from female, but not male, donors. Am J Physiol Lung Cell Mol Physiol, 2016. 310(5): p. L415-25.

65. Furusyo, N., et al., Raloxifene hydrochloride is an adjuvant antiviral treatment of postmenopausal women with chronic hepatitis C: a randomized trial. J Hepatol, 2012. 57(6): p. 1186-92.

66. Gordon, D.E., et al., A SARS-CoV-2 protein interaction map reveals targets for drug repurposing. Nature, 2020. 583(7816): p. 459-468.

67. Caruso, A., et al., Methotrexate inhibits SARS-CoV-2 virus replication "in vitro". J Med Virol, 2021. 93(3): p. 1780-1785.

68. Caccuri, F., et al., A persistently replicating SARS-CoV-2 variant derived from an asymptomatic individual. J Transl Med, 2020. 18(1): p. 362. 
69. Wang, M., et al., Remdesivir and chloroquine effectively inhibit the recently emerged novel coronavirus (2019-nCoV) in vitro. Cell Res, 2020. 30(3): p. 269-271.

70. Caccuri, F., et al., Temporal viral loads in respiratory and gastrointestinal tract and serum antibody responses during SARS-CoV-2 infection in an Italian pediatric cohort. Clin Immunol, 2021. 225: p. 108695.

71. Yuan, S., et al., Metallodrug ranitidine bismuth citrate suppresses SARS-CoV-2 replication and relieves virus-associated pneumonia in Syrian hamsters. Nat Microbiol, 2020. 5(11): p. 1439-1448.

72. Aziz, M., R. Fatima, and R. Assaly, Elevated interleukin-6 and severe COVID-19: A meta-analysis. J Med Virol, 2020. 92(11): p. 2283-2285.

73. Ozmen, B., et al., Influence of the selective oestrogen receptor modulator (raloxifene hydrochloride) on IL-6, TNF-alpha, TGF-beta1 and bone turnover markers in the treatment of postmenopausal osteoporosis. Eur Cytokine Netw, 2007. 18(3): p. 148-53.

74. Rahimian, R., et al., Raloxifene enhances nitric oxide release in rat aorta via increasing endothelial nitric oxide mRNA expression. Eur J Pharmacol, 2002. 434(3): p. 141-9.

75. Morrell, E.D., et al., Experimental therapies for hypoxia-induced pulmonary hypertension during acute lung injury. Shock, 2006. 25(3): p. 214-26.

76. Qi, D., et al., Omentin protects against LPS-induced ARDS through suppressing pulmonary inflammation and promoting endothelial barrier via an Akt/eNOS-dependent mechanism. Cell Death Dis, 2016. 7(9): p. e2360.

77. Kouznetsova, J., et al., Identification of 53 compounds that block Ebola virus-like particle entry via a repurposing screen of approved drugs. Emerg Microbes Infect, 2014. 3(12): p. e84.

78. Gillim-Ross, L., et al., Discovery of novel human and animal cells infected by the severe acute respiratory syndrome coronavirus by replication-specific multiplex reverse transcription-PCR. J Clin Microbiol, 2004. 42(7): p. 3196-206.

79. Matsuyama, S., et al., Enhanced isolation of SARS-CoV-2 by TMPRSS2-expressing cells. Proc Natl Acad Sci U S A, 2020. 117(13): p. 7001-7003.

80. Tseng, C.T., et al., Apical entry and release of severe acute respiratory syndrome-associated coronavirus in polarized Calu-3 lung epithelial cells. J Virol, 2005. 79(15): p. 9470-9.

81. Mossel, E.C., et al., Exogenous ACE2 expression allows refractory cell lines to support severe acute respiratory syndrome coronavirus replication. J Virol, 2005. 79(6): p. 3846-50.

82. Kaye, M., SARS-associated coronavirus replication in cell lines. Emerg Infect Dis, 2006. 12(1): p. 12833.

83. Zhou, P., et al., A pneumonia outbreak associated with a new coronavirus of probable bat origin. Nature, 2020. 579(7798): p. 270-273.

84. Sims, A.C., et al., SARS-CoV replication and pathogenesis in an in vitro model of the human conducting airway epithelium. Virus Res, 2008. 133(1): p. 33-44.

85. Tsuchiya, Y., et al., Antiviral activity of natural occurring flavonoids in vitro. Chem Pharm Bull (Tokyo), 1985. 33(9): p. 3881-6.

86. Verma, S., et al., Anti-SARS-CoV Natural Products With the Potential to Inhibit SARS-CoV-2 (COVID19). Front Pharmacol, 2020. 11: p. 561334.

87. Wei, Y., et al., Antiviral Effects of Novel 2-Benzoxyl-Phenylpyridine Derivatives. Molecules, 2020. 25(6).

88. Dickler, M.N. and L. Norton, The MORE trial: multiple outcomes for raloxifene evaluation--breast cancer as a secondary end point: implications for prevention. Ann N Y Acad Sci, 2001. 949: p. 13442.

89. Barrett-Connor, E., et al., Effects of raloxifene on cardiovascular events and breast cancer in postmenopausal women. N Engl J Med, 2006. 355(2): p. 125-37.

90. Vogel, V.G., The NSABP Study of Tamoxifen and Raloxifene (STAR) trial. Expert Rev Anticancer Ther, 2009. 9(1): p. 51-60.

91. Montopoli, M., et al., Clinical outcome of SARS-CoV-2 infection in breast and ovarian cancer patients who underwent antiestrogenic therapy. Ann Oncol, 2021. 32(5): p. 676-677. 